\title{
A Social Framework for Set Recommendation in Group Recommender Systems
}

\author{
Lorena Recalde
}

\begin{abstract}
This research article presents a study about the background in Group Recommender Systems and how social factors are directly related to these applications. Some important group recommender systems in academia are described to exemplify their contribution in different domains. Besides, a framework that is intended to improve group recommender systems is proposed. The main idea of the framework is to enhance social cognition to help the group members agree and make a decision. Its structure includes a process where an influential group is detected among the target groups of people to recommend to. Social influence detection uses the knowledge behind online social connections and interactions. Trying to understand human behavior and ties among groups in a social network and how to use this to improve group recommender systems is considered the main challenge for future research. Combining this with the kind of item recommendation which involves a temporal sequence of ordered elements will present a novel and original path in Group Recommender Systems design.
\end{abstract}

Index Terms - group preferences, group recommender systems, information propagation, social factors, social attraction.

\section{INTRODUCTION}

$\mathrm{T}$ HE adaptive web provides information sites where the users can be benefited from high degrees of personalization. For instance, e-commerce offers certain products to a specific user who actually needs or likes them, and a video player website profiles the users to extract the list of videos to present particularly to each of them. Today, online social networks customize the user's contact updates board depending on which of his/her friends the user is interested in knowing more about. Most of these websites have as part of their implementation a recommender system. For example, websites like Amazon, Netflix, Last.fm, Pandora, YouTube, etc. incorporate a recommender engine. The recommender is responsible for building the user interests model and finding the item or ranked list of items that best fits their preferences and needs. Therefore, the level of personalization increases when the recommender system knows more about the user.

The target user might be a single user or a group of people. Thus, considering the type of target user, the recommenders are classified in Recommender Systems and Group Recommender Systems respectively. This classification has been proposed since modeling the interests of a person is not the same as modeling the interests of a family, a sport team, a group of friends or a group of people who are sharing a room.

Particularly, the present article focuses on the analysis of literature in group recommender systems and the social factors involved in order to propose a framework that models the research findings in a way that improvements may be added. Group recommenders must be able to identify items that the group of users will like so that their needs, explicit or not, are equally satisfied. It can be implemented considering three different components: i) the nature of the target group to recommend to, ii) the kind of recommendation made (one item, an ordered set of elements, a bunch of items put together), and iii) external factors (groups dynamics, social influence, personality, tie strength and emotions) that may be considered when formulating the recommendation techniques used to match group - items.

Group recommender systems are relevant because activities like watching movies, eating in restaurants and having holiday trips are usually done by groups. Their main aim is to augment social cognition. As a result, the group members use the recommender because it is easy for them to agree and make a decision, it enhances the members' participation, it provides them with a strong sense of belonging and it offers reliable suggestions.

With the wide development and expansive use of Online Social Networks, researchers have realized that the technical and visual requirements that satisfied people's needs are not sufficient any more. Human Computer Interaction studies have gone further in order to analyze, prototype and evaluate society or community needs. The Social Web does not imply people interacting with a machine. It represents people interacting with people thanks to machines. Consequently, sciences like psychology and sociology play an important role in sociotechnical systems design [1].

Considering the statements mentioned above, and the fact that we interact with people more than we consume a service or a product, it is justified the study of groups dynamics and other social factors in group recommender systems. In consequence, a new - social - approach in group recommenders design will be introduced in this paper, aiming to improve the user experience, from algorithms to interface.

Questions to guide the research are:

- Can groups of people be influenced by other groups at the moment they are making a decision? If yes, how to implement this social factor in a Group Recommender System? - Should susceptible groups' preferences be model in a different 
way from influential groups model?

- Is it important to let susceptible groups visualize the influential group members, their choice and the reasons why they made that decision? Does it help or manipulate them?

The article is structured as follows. Section II details the context of the article and the state of the art in group recommender systems, the kinds of domains and approaches implemented as well as the social factors studied in recommenders. The social framework proposed and the methodology to embed it in a group recommender system is presented in Section III. Section IV presents the current challenges by associating the concepts and techniques, as well as the fundamental issues in recommender systems to the research that is needed for future work. To finish, Section V presents the conclusions of the article.

\section{Context And State OF THE ART}

In the light of the above overview about the main function of a recommender system, we could say that the popularity of those systems has increased because of their usefulness. In fact, there are three relevant reasons that justify the importance of those systems in the people's daily lives: i) we make choices about every aspect that is part of our lives all the time [2]; ii)the quantity of available online information about different alternatives, services or products is constantly increasing, so we have to rely on others' opinions and recommendations to make good decisions; and iii) computational systems were created with the aim of augmenting human cognition, so people can remember, think and reason in better ways [3]. Therefore, it is important to have systems that support the decision-making process.

The term item is a general word used to make reference to the object that the recommender system suggests. Accordingly, an item to recommend would be a singer, a movie, a restaurant, a Twitter user to follow or a Facebook friend to add. However, the recommendation might be not only a ranked list of independent items, from which the user selects, buys or adopts any of the items presented. It may be an ordered set of elements, where one item recommendation signifies some elements provided in a specific order, or a bunch of items put together having the notion of better together, so a bundle of two or more objects conforms the item recommendation [4]. The nature of the recommendation or the type of item recommended is usually determined by the system domain. The domain guides the design of the recommender system because the approaches and techniques to implement may differ depending on whether the system recommends a recipe, a medical treatment or a car to rent.

The approaches applied in recommender systems have evolved since mid-1990's. Many improvements to the algorithms and techniques have been published as a result of academia and industry research. The main approaches are:

- Collaborative Filtering. The algorithms use historical rating information to compare how similar the users' preferences are. The search of neighbors of the current user allows to recommend him/her items with high ratings provided by his/her peers.

- Content-based techniques. The recommender bases its suggestions on the degree of high previous acceptance of items which have the same features or attributes as the ones unseen by the user. Therefore, because of their similarity they may be recommended.

- Knowledge-based techniques. In these systems, there are knowledge bases about users and items. Most of the time the needs are elicited through conversational interactions between the user and a recommender assistant until discovering the item that has the desired characteristics.

The approaches mentioned have different variations and may be combined as a hybrid recommender system [5] in order to minimize their individual drawbacks. In recent years ContextAware, Social-Based and Trust-Aware Recommenders have also emerged to present paradigms that the recommender systems developers may analyze to find which of the approaches best suits the requirements of the system.

Decisions about the design of the recommender have to be made after knowing the item to recommend or, in other words, once the domain is defined. However, knowing which target user to recommend to has the same importance. Group preferences modeling is a demanding task and it differs from single users modeling process. This section presents the previous work on group recommender systems, different kinds of items recommendation: single object, bunch of elements as well as temporal sequence of items, and social factors in the recommendation process. A summary with relevant information is detailed in Table I.

\section{A. Group Recommender Systems}

In context aware recommendations the system has to evaluate the present condition of the user, taking into account, for example, the localization, time, weather and company [6]. For instance, the idea of such a recommender system is to consider the preferences of the user, but when he or she is in the company of friends it should change the context of the user to adapt the recommendation for a group of people. Nevertheless, it is not a group recommender system. A group recommender system supports the recommendation process by modeling the preferences of a group of people generally, by using aggregation methods. This is needed when there is an activity (domain) that can be done or enjoyed as a group.

The aggregation methods to extract the group's interests to build the group's model work on combining the previous ratings of the individuals into a single group rating. In [7], Masthoff presents the evaluation of aggregation methods in an Interactive Television recommender system. She chose that domain considering that watching $\mathrm{TV}$ is the most frequent activity done in family and that the group of people usually have heterogeneous preferences. Her work presents the results obtained after studying how some aggregation strategies work, such as multiplicative, approval voting, least misery, most pleasure, fairness, and so on. She found that average, average without misery, and least misery are good candidates for implementation. Some aggregation methods and other ways to generate group recommendations are described with examples in [8].

One of the first group recommender systems was presented in [9]. Here the authors developed MusicFX, a recommender 
TABLE I

GROUP RECOMMENDER SYSTEMS APPLICATIONS: EXAMPLES IN DIVERSE DOMAINS.

\begin{tabular}{|c|c|c|c|c|c|}
\hline Interactive Television & tv programs & temporal sequence & family & framework: explicit elicitation & $\begin{array}{l}\text { affective state after watching a } \\
\text { program }\end{array}$ \\
\hline MusicFX & music station & one item & $\begin{array}{l}\text { people working } \\
\text { out in a gym }\end{array}$ & $\begin{array}{l}\text { explicit elicitation of music genre } \\
\text { preferences }\end{array}$ & rating scale $-2,-1,0,1,2$ \\
\hline INTRIGUE & $\begin{array}{l}\text { tourist } \\
\text { destinations }\end{array}$ & temporal sequence & family & $\begin{array}{l}\text { explicit elicitation of preferences + } \\
\text { socio-demographic information }\end{array}$ & $\begin{array}{l}\text { contextual information: } \\
\text { geolocation and schedules of } \\
\text { activities }\end{array}$ \\
\hline $\begin{array}{l}\text { Poker Restaurant } \\
\text { Finder }\end{array}$ & restaurants & ranked list & any group & $\begin{array}{l}\text { explicit elicitation of preferences } \\
\text { and needs priority classification }\end{array}$ & $\begin{array}{l}\text { context information: current } \\
\text { place of group }\end{array}$ \\
\hline
\end{tabular}

that presents a music station to play for the group of people working out in a gym. The article states that an intelligent environment (a fitness center, a restaurant or a store) should respond to the group of people who are the current inhabitants by recognizing who they are and what preferences they have.

Therefore, the system can play the music the clients like. MusicFX obtains the information about the clients' interests from a database that stores their music genre preferences (previously and explicitly specified). With the use of an authentication mechanism, the system controls who are the clients that are in the gym at a given time. After applying an aggregation algorithm, the system computes the group ranking for each music genre, so it randomly chooses which music station to play among the top $\mathrm{n}$ ranked stations. ${ }^{1}$

The research work presented in [10] shows another group recommender system, INTRIGUE. This recommender was designed to offer personalized suggestions about tourist environs in a specific geographical area to constrain the location for the tour. It recommends multiple destinations to visit and itineraries considering the preferences of groups, such as families with heterogeneous kinds of members like children and elderly. The aggregation method differs from the one used in Interactive Television and MusicFX where the aggregation is done by extracting the individual preferences to finally have the group's interests model to be able to compute the recommendation. On the other hand, in INTRIGUE the individual recommendations for each member or for homogeneous subgroups are computed, and then they are aggregated to have the entire group's recommendation. ${ }^{2}$ The system applies a variation of the average aggregation strategy.
The weights depend on the size of the homogeneous subgroups and their relevance. That is to say, if there is a subgroup of children, they are more relevant or their recommendation weighs more when computing the whole group recommendation.

Similarly, in [12], McCarthy proposes the Pocket RestaurantFinder, that recommends restaurants to groups of people considering their culinary preferences and location. Specifically, the recommender uses information like travel distance, expected facilities, cuisine desired and budget planned. When using the recommender system, the group members have to express explicitly and individually the desired values for the four features, and they also need to order the features in a level of priority. Then, Pocket RestaurantFinder computes the recommendation by applying an average preferences aggregation method. The restaurants are displayed in a ranked list that matches the group's likes.

The system in [13] recommends web pages by exploring the implicit behavior of communities of searchers, where a community is defined as a group of users with similar information needs. The authors argue that if there are users with very similar information requirements, they send similar queries to the search engine. For the system, named I-SPY, it is important to extract the user preferences by considering the query repetition and selection regularity (which pages they click among the retrieved ones) measures in web search. If the search activity is performed within a well-defined context, let's say in a specific website search box, the set or community of users are known to have specific information preferences. As the community uses the search engine, the system will gradually

${ }^{1}$ There are 91 stations and each one is associated with a music genre.

${ }^{2}$ Recommendation aggregation or merging was also considered to be used in the PolyLens recommender [11], but for the domain this method presented significant drawbacks. 
adapt the ranking pages function considering the historical data about a given query and the clicked results for it.

In [14], Jameson et al. detail the prototype of the group recommender Travel Decision Forum. The web-based system is designed to recommend places for vacation to some friends who will perform an asynchronous communication through the system in order to agree. The prototype makes use of an animated character who shows the potential trip options and plays the role of mediator. Its role is to help the group make a decision. The preferences concerning the vacation need to be expressed individually by every person in the group. Then, the recommender uses a preferences aggregation method to define the group's preferences as a whole. However, an important issue to be solved in the Travel Decision Forum is to allow each member to be aware, so every group member can visualize the others' preferences in the interface.

I have presented some group recommender systems and how they perform the recommendations in different application domains. Research works like [8], [15] and [16] detail more group recommenders, kinds of target groups, methods to compute the group recommendations and some explanations about the group recommendation process design. The authors agree that the existing challenges in group recommender systems are not similar to those seen in common recommender systems.

In fact, the problems that arise are harder to solve. In their works, they emphasize how important it is to do more research in social issues such as influence among the members and their attitude when deciding about an alternative, the affective state of the members of the group while enjoying a set of activities, and the nature of groups formed. For example, they might be an established, an occasional or a random group.

As was mentioned before, it is necessary to know what the recommendation is going to be. Consequently, the next section reviews the literature about the nature of the items to recommend.

\section{B. Items Recommendation}

The state of the art in recommender systems is very broad. However, it usually addresses the analysis and improvement of approaches like Collaborative Filtering [17], Content-Based [18], Constraint-Based [19] and Hybrid Recommender Systems [20] considering individual item recommendation to single users. The recommendation of items is generally presented as a ranked list of individual objects and the user can choose one item or another because they are independent. The web-based recommender system Movielens (www.movielens.umn.edu) [21], uses the collaborative filtering approach to predict the user rating for a movie. A set of ratings on already seen movies has to be provided by the user. Consequently, the system can recommend movies whose rating value predictions are high for the user.

In [22], a content-based recommender system, called Informed, is explained. The system creates an ontology for the items based on the previous consumer reviews. Natural Language Processing techniques and Text mining are applied to extract the features or attributes of the item and identify each of them as good or bad, according to users' opinions. For example, a photo camera may have good resolution and bad battery life. The system will give a weight for the features depending not only on the quality feature classification, but also on the degree of relevance of the feature for the user. The Informed system uses the expertise information about the user to compute the weights and then produces a ranked list of items that best suits his/her needs.

The research done in [23], shows a content-based movie recommender called Cinemappy. The application works in mobile devices and uses data extracted from DBpedia about each of the movies, as well as contextual information related to the current time and location of the user. The computation of similarity between movies is done taking into account shared features like same director, same genre, same stars, for example. Similarity helps to identify other movies that the user will like because they have common features with previously seen movies which the user Liked. Google Places and Trovacinema are websites which use available information to extract the contextual data. For example, Cinemappy will recommend a list of movies, including information like their genres and the cinema name where the movie is showing. Additionally, it will let the user know the distance from his/her current geographical location to the cinema, which is a constraint to produce the recommendation. However, the system can also show other cinemas that play the movie chosen by the user.

Little work has been done when a single recommendation is composed by some units, ordered or not. For example, in [24] the system creates a playlist of songs for the user. It is not static, so if a new song appears the playlist is reorganized considering the user model and then the personal recommendation of the new arranged set of songs is made. In this kind of recommenders, when the suggestion is made up of a sequence of elements, most of the time their position depends on the user interests or other constraints. Consequently, at the moment the user chooses an item, he/she has access to a set of ordered units. In [25] another similar system, patented by Amazon Technologies, is detailed. The algorithm output presents three or more items that work well together, so they are recommended as a bundle (in this case, without a specific order), refining the idea of better together.

The systems previously mentioned were developed taking into account that the target user is a single one. ${ }^{3}$ Nevertheless, two of the group recommender systems studied in the preceding section show interesting items recommendation. For instance, the Interactive Television system [7] presents an ordered sequence of TV programs. The order is assigned by considering the preferences of the group and their affective state after having seen a program. The idea is to balance the satisfaction of the group members by ordering the programs

\footnotetext{
${ }^{3}$ Cinemappi is a recommender that handles contextual information, so the user has the possibility to tell the system that he/she wants to watch a movie with a friend; however, the system does not model the preferences of groups.
} 
correctly. INTRIGUE [10] recommends multiple attractions to be visited by the group during their trip. It considers a sequence of places in the recommendation without any other restriction than the visits schedule.

We have seen important research works related to the most relevant issues in recommender systems: users (single users versus groups) and items (lists of individual items, bundles of items and sequences). Table I presents a summary of that information considering group recommender system examples.

Next, studies about social factors in the recommendation process are detailed.

\section{Social Factors in Recommender Systems}

Understanding the user's interests and needs is not enough to design a recommender system that takes into account the user experience. Visualization techniques, human cognition, social behavior, choice theory, persuasion, information diffusion and community formation are some of the concerns when implementing an application for the Web. In fact, social recommender systems or recommenders for the Social Web have emerged with the aim of modeling the user's preferences by using the information he or she and their friends have published in online social networks [26].

In [27] the authors propose a framework to merge behavioral theory and social recommender systems design. They make their proposal based on the argument that social and psychological theories may be employed as sources of Information Systems design principles. Therefore, in the authors' studies it is proved that homophily, tie strength, and trustworthiness leverage the recommendation acceptance (sociological view).

The researchers in [28], also model the preferences of the user in a social recommender, but they take into account that some of the user's friends might have different interests. Their argument is that we always look for our friends' recommendations, so in their work they establish the difference between trust relationships and social friendships. In a social network, a trust relationship is understood as users who may not know each other and there are unilateral connections. However, a social friendship reflects real and mutual relationships, so this kind of links are the important ones when implementing a social recommender system. In this work, the authors represent the diversity of tastes among the user's social connections (matrix factorization) to improve the accuracy of the recommendations.

In [29], the authors propose an approach for group recommender systems by merging Collaborative Filtering and a Genetic Algorithm that learns from known group ratings. The authors state that important social effects like opinion leadership, influence in thoughts, feelings, and actions as well as kinds of interactions, are present in group decision-making. Therefore, they need to be considered in group recommender systems. Actually, the social factor included in the framework proposed is the preceding interaction among group members reflected in their past ratings as an individual and also as subgroups. An interesting metric for the system is influential personality, that can be inferred from the ratings.

In [30], Quijano et al. study individual behaviors, group personality composition and trust relationships among members of the group to make recommendations for them. They propose a set of methods that could be integrated into any social network and then make recommendations like movies, restaurants, trips, etc. The system is able to infer social characteristics about the group members. For example, the approach evaluates parameters like collaborating, consensuating, evasion, and may assume a permissiveness personality; another parameter may be closeness between friends. Having these values, the system improves the group decision-making process.

Other recent works in group recommender systems have tested the way the recommendations are presented in the interface in order to prove that showing members emotions about the item can influence the user adoption. For instance, in [31], Chen et al. show that the integration of emotion cues in GroupFun, a recommender mobile system that suggests songs for a group of friends, might make each of them be aware of the others' preferences. The system generates playlists considering the aggregation of the members' ratings, but mutual awareness may influence the rating values when a user sees how his/her friends feel about the song.

\section{SOCIAL FRAMEWORK FOR GROUP RECOMMENDERS}

The wide and quick spread of the use of online social networks is an evidence that the users not only need to contact other users and establish social connections, but also that they look for information generated by other people around. Therefore, a way to keep the social links with others is being aware of what they do, think, feel, share and buy. On one hand, human factors influence a person's decision. In fact, literature about personalization improvement in the recommendation process has shown that including personality as well as cognitive and learning style has a positive impact on the extraction of long-term preferences to design the user preferences model [32]. On the other hand, a user who has his online profile is able to interact with a huge number of people, and social factors arise showing that only some of his contacts influence his decisions, enrich his reasoning skills and provide additional knowledge through their online activity. Actually, the degree of social connections influence does not depend on the user personality, but on his desire to belong to a community.

This paper proposes a new recommendation framework to include information about influential groups' decisions (in the preferences model adaptation and in the interface) in a system where the target is a group of people and the recommendation is a sequence of ordered elements.

The research questions presented in Section I plus the current state of research seen in Section II are considered as guidelines to define the main components in the framework proposed (Figure 1).

\section{A. Influential Group Identification}

The detection of the influential group among the target groups to which recommend needs to implement both: the recognition of the groups' members who are known because of 


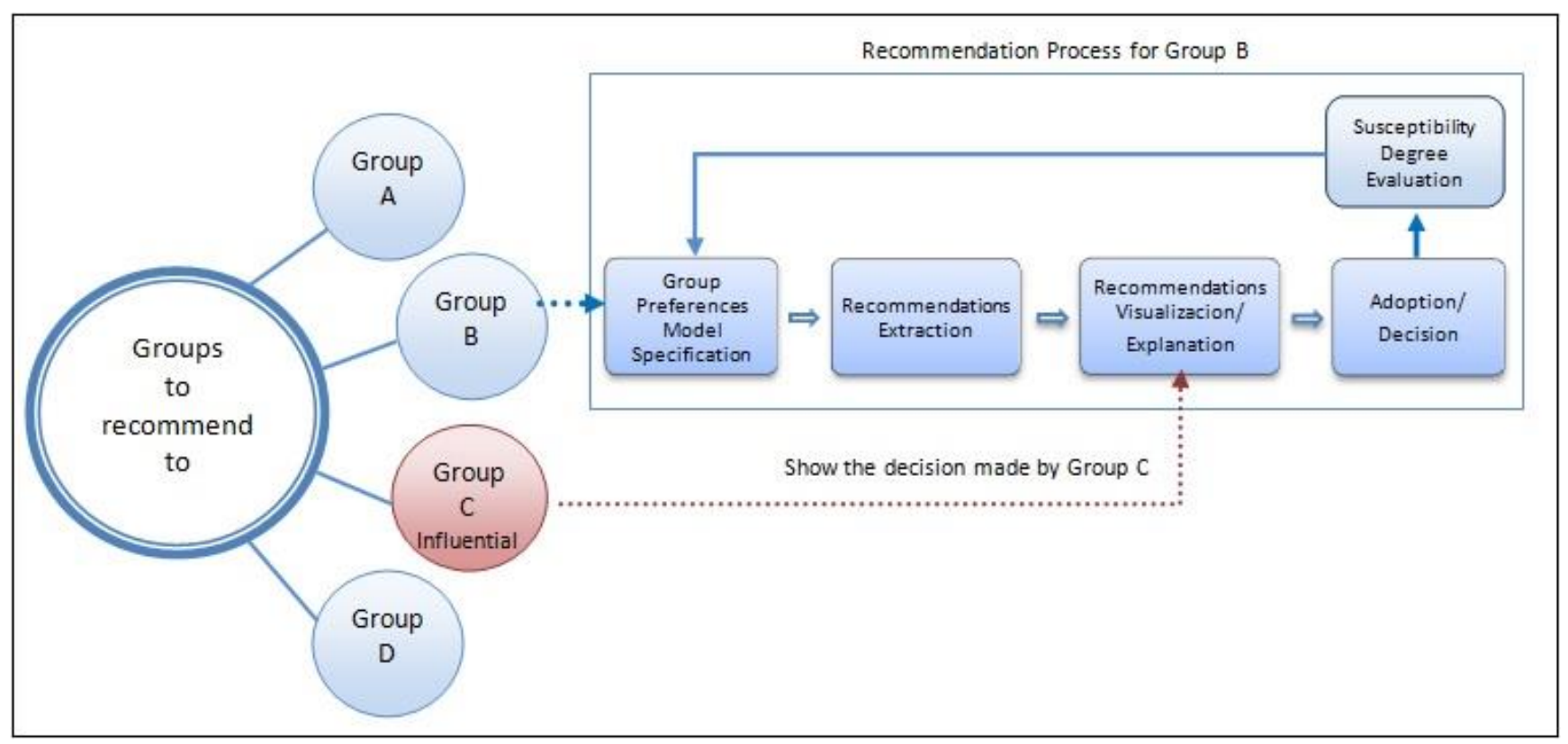

Fig. 1. After identifying the influential group/s and knowing its decision, the recommendation process for the Group B will let them know which was the choice of the influential group and why they chose that option. If group B decides to make the same choice, the model of Group B preferences will adapt his level of susceptibility.

their expertise background, sensibleness, trust and extroversion and information diffusion through communities by mining online social networks. Once having this information processed the extraction of main influential groups could be possible, presenting also the rest of groups that are susceptible to easily adopt a recommendation made by an influential group.

\section{B. Group Preferences Model and Adaptation}

What defines a group of people is their similarities, so that they could recognize the social category they belong to, and also the social categories they do not. A group has its social identity established when the members see themselves as a group. Self categorization theory says that when a person sets the differential parameters with other individuals, he sees himself with his own identity; on the other hand, when he is aware that he has a membership in a group he maximizes perceptually his similarities with the rest of members reducing in this way, their individual differences [33]. This fact will be considered at the moment of formulating the preferences aggregation method: the extraction of individual interests has a lower impact than the rate of items experienced before for the group as a whole, its current expectations, present goals and needs. The model should define the group identity in contrast to other groups. Actually, an influential group has a preferences model that includes different parameters than the susceptible groups, which have a model that adapts the parameter of susceptibility every time that they choose the influential group recommendation.

\section{Sequence of items prediction}

The kind of recommendation is planned to be a sequence of elements order in a way that all the group members enjoy the social activity. For example, the recommendation for group A

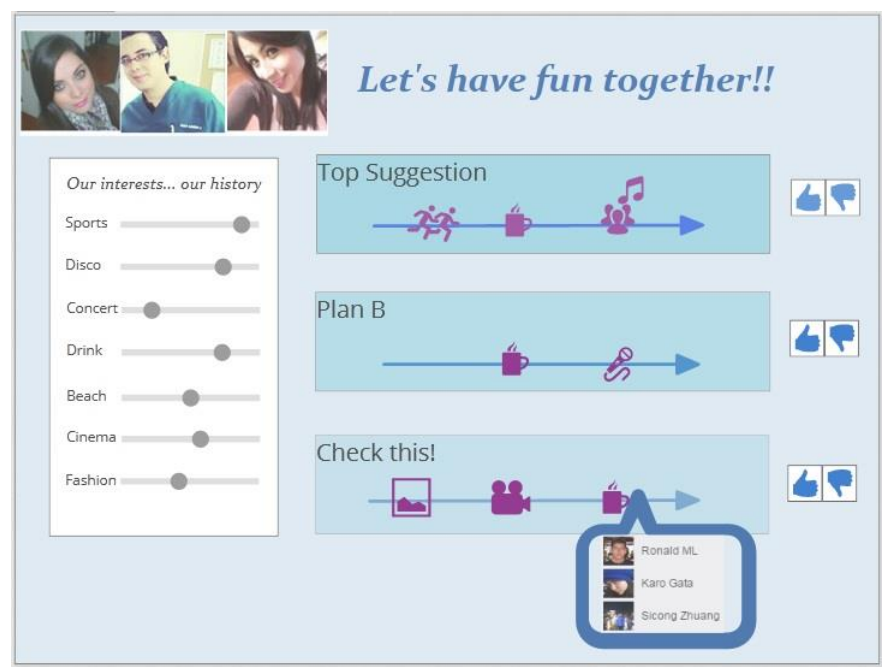

Fig. 2. Features to be present in the Group Recommender Interface.

could be: element $\mathrm{p}$, then element $\mathrm{q}$ and them element $\mathrm{r}$; while for group B it is: element $p$, element $o$ and then element $r$. In fact, the preferences model of the group expresses the features needed or desired and the sequential integration of the elements recommended in a specific order should match those group needs. Generally, the approaches used depend on the domain of the recommendation: entertainment, content, e-commerce, service or social item. In the scope of the present research, the recommender system is thought to suggest leisure activities for a weekend with family or friends. That is to say, a sequence of leisure events which order is based not only in contextual information, but also in the preferences model and the estimated degree of acceptance of the recommendation of the influential group adoption. 
TABLE II

METHODOLOGY ACCORDING TO THE FRAMEWORK COMPONENTS

\begin{tabular}{|c|c|}
\hline Framework Component & Methodology \\
\hline \multirow{2}{*}{$\begin{array}{l}\text { Influential Group } \\
\text { Identification }\end{array}$} & $\begin{array}{l}\text { Social Web Mining as well as Big Data and } \\
\text { Information Diffusion Analysis }\end{array}$ \\
\hline & $\begin{array}{l}\text { Comparison Social Theories vs User } \\
\text { studies and Log Data Analysis }\end{array}$ \\
\hline \multirow{4}{*}{$\begin{array}{l}\text { Preferences Model and } \\
\text { Adaptation }\end{array}$} & $\begin{array}{l}\text { Social Web Mining and NLP (Sentiment } \\
\text { Analysis) }\end{array}$ \\
\hline & Preferences Elicitation Techniques \\
\hline & IR techniques \\
\hline & $\begin{array}{l}\text { Preferences Aggregation and methods to } \\
\text { include a Susceptibility Adaptation } \\
\text { parameter }\end{array}$ \\
\hline \multirow{4}{*}{$\begin{array}{l}\text { Sequence of Items } \\
\text { Prediction }\end{array}$} & Contextual information extraction \\
\hline & IR techniques \\
\hline & $\begin{array}{l}\text { User studies to evaluate order of } \\
\text { elements }\end{array}$ \\
\hline & $\begin{array}{l}\text { Evaluation of the accuracy of the } \\
\text { prediction }\end{array}$ \\
\hline \multirow{3}{*}{$\begin{array}{l}\text { Recommendations } \\
\text { Visualization }\end{array}$} & High-Fidelity Prototyping \\
\hline & Usability tests \\
\hline & User Centered Design Techniques \\
\hline
\end{tabular}

\section{Recommendations Visualization}

The goal of the Group Recommender System Interface is to support cooperative work in a way that the members of the group can be aware of one another needs but still they can see themselves as a whole, who have a common aim. Its design will be centered in characterizing the group interests and offer the option to see why one group they know (the influential one) chose a specific recommendation so that they could trust this is a good recommendation also for them (Figure 2). In this way, the interface pretends to implement a conflict resolution feature to help, in a non intrusive way, the group to make a decision faster. The recommender engine is half of the system; the other half is having groups using it to find the social activity that better matches their preferences.

The components of the framework discussed above require a methodology summarized in Table II.

\section{Challenges AND Future Work}

The state of the art review has described illustrative approaches, frameworks and systems that represent fundamental research in recommender systems. Nevertheless, it has been shown that there are social requirements that need to be addressed specifically in group recommender systems. Business logic as well as social factors have to be included in recommender algorithms and they also have to integrate ways to give importance to the user experience [34]. There are some challenges facing current concepts and techniques:

- Target User: group of people with heterogeneous interests, size evaluation.
- Nature of the Recommendation: algorithms to suggest a set of items in a sequential order depending on certain context to improve the process.

- Social Factors: Analysis about human behavior (emotions, personality, social identity, awareness) considering the inter-group level of interaction, as well as social behavior (influence, collaboration, curiosity) in the intra- group level.

- Group preferences model: scheme that evaluates preferences aggregation methods and inclusion of historical group ratings.

- User Experience: UX is designed in every phase of the recommendation, from algorithms to visualization.

- Interface: display of other groups' choices and explanations to facilitate agreement among the group.

The combination of those features is a novel approach that, if the pieces fit well, could improve group recommender systems. For future work, the implementation of the social framework shown in Section III in a group recommender system is planned. Its evaluation will be carried out assessing the four components: Influential Group Identification, Group Preferences Model and Adaptation, Sequence of items prediction and Recommendations Visualization.

\section{CONCLUSIONS}

Previously, I made evident the effort that has been invested in studying social factors to improve recommender systems for single users or groups. Nevertheless, group recommender systems research is scarce compared to the great improvements found in personal recommender systems. The assumptions that explain the reasons are:

- Recommender systems need users' information as input to build the user interests model. This information can be gathered by processing the explicit actions of the users (ratings, opinions, purchases) and/or their implicit feedback (search queries, item navigation through, clicks) [4]. There are datasets with this information for the Web activity of single users, but it may be a challenge to find datasets in academia about ratings given by groups as a whole [35].

- By mining online social networks it is possible to analyze the users' behavior and know who are influential persons [36]. On the other hand, it is needed to make studies about detecting influential communities in the Social Web but that can be applied in a group recommender system.

- Social factors have been investigated in group recommenders by taking into consideration the intra-group level (between the group members) but not in the intergroup level (among groups) because there has been a gap separating sociological theories and computer science until the Social Web arrival. Each framework component faces specific challenges and needs to be implemented by defining its own methodology and techniques. Some of them will have psychological and sociological information as input, others will need to be tested by applying more than one approach and algorithm combinations. 
Understanding the nature of a group, their dynamics, how they are formed, size of influential groups and the ways they interact by using online social networks is the first issue to address.

\section{REFERENCES}

[1] B. Whitworth and A. Ahmad, The Social Design of Technical Systems: Building technologies for communities, 2nd ed. Aarhus C, Denmark: The Interaction Design Foundation, 2014.

[2] P. Resnick and H. R. Varian, "Recommender systems," Commun. ACM, vol. 40, no. 3, pp. 56-58, Mar. 1997.

[3] E. H. Chi, "The social web: Research and opportunities," Computer, vol. 41, no. 9, pp. 88-91, 2008.

[4] F. Ricci, L. Rokach, and B. Shapira, "Introduction to recommender systems handbook," in Recommender Systems Handbook, F. Ricci, L. Rokach, B. Shapira, and P. B. Kantor, Eds. Springer US, 2011, pp. 1-35.

[5] R. Burke, "Hybrid web recommender systems," in The Adaptive Web, ser. Lecture Notes in Computer Science, P. Brusilovsky, A. Kobsa, and W. Nejdl, Eds. Springer Berlin Heidelberg, 2007, vol. 4321, pp. 377- 408.

[6] G. Adomavicius and A. Tuzhilin, "Context-aware recommender systems," in Recommender Systems Handbook. Springer US, 2011, pp. 217-253.

[7] J. Masthoff, "Group modeling: Selecting a sequence of television items to suit a group of viewers," in Personalized Digital Television, ser. Human-Computer Interaction Series. Springer Netherlands, 2004, vol. 6, pp. 93-141.

[8] A. Jameson and B. Smyth, "Recommendation to groups," in The Adaptive $W e b$, ser. Lecture Notes in Computer Science. Springer Berlin Heidelberg, 2007, vol. 4321, pp. 596-627.

[9] J. F. McCarthy and T. D. Anagnost, "Musicfx: An arbiter of group preferences for computer supported collaborative workouts," in Proceedings of the 1998 ACM Conference on Computer Supported Cooperative Work, ser. CSCW '98. New York, NY, USA: ACM, 1998, pp. 363-372.

[10] L. Ardissono, A. Goy, G. Petrone, M. Segnan, and P. Torasso, "Intrigue: Personalized recommendation of tourist attractions for desktop and hand held devices," Applied Artificial Intelligence, vol. 17, no. 8-9, pp. 687714, 2003.

[11] M. OConnor, D. Cosley, J. Konstan, and J. Riedl, "Polylens: A recommender system for groups of users," in ECSCW 2001. Springer Netherlands, 2001, pp. 199-218.

[12] J. F. McCarthy, "Pocket restaurant finder: A situated recommender system for groups," in Proceeding of Workshop on Mobile Ad-Hoc Communication at the 2002 ACM Conference on Human Factors in Computer Systems, 2002.

[13] B. Smyth, E. Balfe, J. Freyne, P. Briggs, M. Coyle, and O. Boydell, "Exploiting query repetition and regularity in an adaptive communitybased web search engine," User Modeling and User-Adapted Interaction, vol. 14, no. 5, pp. 383-423, Jan. 2005.

[14] A. Jameson, S. Baldes, and T. Kleinbauer, "Enhancing mutual awareness in group recommender systems," in Proceedings of the IJCAI 2003 Workshop on Intelligent Techniques for Web Personalization, B. Mobasher and S. S. Anand, Eds. Menlo Park, CA: AAAI, 2003.

[15] J. Masthoff, "Group recommender systems: Combining individual models," in Recommender Systems Handbook. Springer US, 2011, pp. $677-702$

[16] L. Boratto and S. Carta, "State-of-the-art in group recommendation and new approaches for automatic identification of groups," in Information Retrieval and Mining in Distributed Environments, ser. Studies in Computational Intelligence. Springer Berlin Heidelberg, 2011, vol. 324, pp. $1-20$.

[17] B. Sarwar, K. G., K. J., and R. J., "Item-based collaborative filtering recommendation algorithms," 2001, pp. 285-295.

[18] M. Pazzani and D. Billsus, "Content-based recommendation systems," in The Adaptive Web, ser. Lecture Notes in Computer Science. Springer Berlin Heidelberg, 2007, vol. 4321, pp. 325-341.
[19] A. Felfernig and R. Burke, "Constraint-based recommender systems: Technologies and research issues," in Proceedings of the 10th International Conference on Electronic Commerce, ser. ICEC '08. New York, USA: ACM, 2008, pp. 1-10.

[20] R. Burke, "Hybrid recommender systems: Survey and experiments," User Modeling and User-Adapted Interaction, vol. 12, no. 4, pp. 331370, 2002.

[21] B. J. Dahlen, J. Konstan, J. Herlocker, N. Good, A. Borchers, and J. Riedl, "Jump-starting movielens: User benefits of starting a collaborative filtering system with "dead date"," 1998.

[22] S. Aciar, D. Zhang, S. Simoff, and J. Debenham, "Informed recommender: Basing recommendations on consumer product reviews," Intelligent Systems, IEEE, vol. 22, no. 3, pp. 39-47, May 2007.

[23] V. C. Ostuni, T. D. Noia, R. Mirizzi, D. Romito, and E. D. Sciascio, "Cinemappy: a context-aware mobile app for movie recommendations boosted by dbpedia," in Proceedings of the International Workshop on Semantic Technologies meet Recommender Systems \& Big Data, Boston, USA, November 11, 2012, 2012, pp. 37-48.

[24] S. Ward, "System and method for creating dynamic playlists," 2003, uS Patent 6,526,411.

[25] G. Chanda, B. Smith, and R. Whitman, "Automated selection of three of more items to recommend as a bundle," 2013, uS Patent 8,438,052.

[26] I. Guy and D. Carmel, "Social recommender systems," in Proceedings of the 20th International Conference Companion on World Wide Web, ser. WWW '11. New York, USA: ACM, 2011, pp. 283-284.

[27] O. Arazy, N. Kumar, and B. Shapira, "A theory-driven design framework for social recommender systems," Journal of the Association for Information Systems, vol. 11, no. 9, pp. 455-490, 2010.

[28] H. Ma, D. Zhou, C. Liu, M. Lyu, and I. King, "Recommender systems with social regularization," in Proceedings of the Fourth ACM International Conference on Web Search and Data Mining, ser. WSDM '11, New York, USA, 2011, pp. 287-296.

[29] Y. Chen, L. Cheng, and C. Chuang, "A group recommendation system with consideration of interactions among group members," Expert Systems with Applications, vol. 34, no. 3, pp. 2082 - 2090, 2008.

[30] L. Quijano-Sanchez, J. Recio-Garcia, B. Diaz-Agudo, and G. JimenezDiaz, "Social factors in group recommender systems," ACM Transactions on Intelligent Systems and Technology, vol. 4, no. 1, pp. 8:1-8:30, Feb. 2013.

[31] Y. Chen, X. Ma, A. Cerezo, and P. Pu, "Empatheticons: Designing emotion awareness tools for group recommenders," in Proceedings of the $X V$ International Conference on Human Computer Interaction, ser. Interaccion '14. New York, NY, USA: ACM, 2014, pp. 16:1-16:8.

[32] R. Hu and P. Pu, "Enhancing collaborative filtering systems with personality information," in Proceedings of the Fifth ACM Conference on Recommender Systems, ser. RecSys '11. New York, USA: ACM, 2011, pp. 197-204.

[33] J. Turner, P. Oakes, S. Haslam, and C. McGarty, "Self and collective: Cognition and social context," Personality and Social Psychology Bulletin, vol. 20, no. 5, pp. 454-463, 1994.

[34] J. Konstanand, J. Riedl, "Recommender systems: from algorithms to user experience," User Modeling and User-Adapted Interaction, vol. 22, no. 1-2, pp. 101-123, 2012.

[35] S. Shang, Y. Hui, P. Hui, P. Cuff, and S. Kulkarni, "Beyond personalization and anonymity: Towards a group-based recommender system," in Proceedings of the 29th Annual ACM Symposium on Applied Computing, ser. SAC '14. New York, NY, USA: ACM, 2014, pp. 266273.

[36] N. B. Ellison, J. Vitak, R. Gray, and C. Lampe, "Cultivating social resources on social network sites: Facebook relationship maintenance behaviors and their role in social capital processes," Journal of Computer-Mediated Communication, vol. 19, no. 4, pp. 855-870, 2014. 


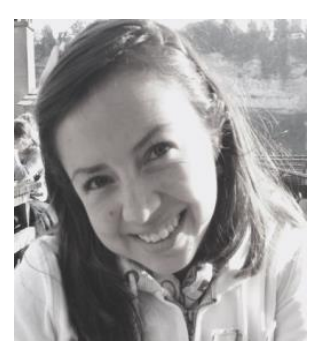

Lorena Recalde received the B.S. degree in computational systems engineering from National Polytechnic School (EPN), Quito, Ecuador, in 2008. From 2010 to 2012 she worked as teacher assistant at the EPN. She received the M.S. degree in computer science from Fribourg University, Fribourg, Switzerland, in 2014. In the same year she started her Ph.D. studies in information and communication technologies at the Pompeu Fabra University, Barcelona, Spain, where currently, she is doing research in the field of Group Recommender Systems. 
